\title{
miR-34a inhibits colon cancer proliferation and metastasis by inhibiting platelet-derived growth factor receptor $\alpha$
}

\author{
CHUNYAN LI ${ }^{1 *}$, YULIN WANG ${ }^{2 *}$, SHUMING LU $^{1}$, ZHUQING ZHANG ${ }^{3}$, \\ HUA MENG $^{1}$, LINA LIANG ${ }^{1}$, YAN ZHANG ${ }^{1}$ and BO SONG ${ }^{4}$ \\ Departments of ${ }^{1}$ Gastroenterology and ${ }^{2}$ General Surgery, The First Affiliated Hospital of Dalian Medical University, Dalian, \\ Liaoning 116011; ${ }^{3}$ Department of Pathology, Dalian Municipal Central Hospital, Dalian, Liaoning 116033; \\ ${ }^{4}$ Department of Pathology, Dalian Medical University, Dalian, Liaoning 116044, P.R. China
}

Received September 15, 2014; Accepted June 22, 2015

DOI: $10.3892 / \mathrm{mmr} .2015 .4263$

\begin{abstract}
The microRNA (miRNA), miR-34a is significant in colon cancer progression. In the present study, the role of miR-34a in colon cancer cell proliferation and metastasis was investigated. It was found that the expression of miR-34a in colon cancer tissues and cell lines was lower when compared with that of normal tissues and cells. Further research demonstrated that miR-34a inhibited cell proliferation, induced $\mathrm{G}_{1}$ phase arrest, and suppressed metastasis and epithelial mesenchymal transition in colon cancer cells. Bioinformatic prediction indicated that platelet-derived growth factor receptor $\alpha$ (PDGFRA) was a potential target gene of miR-34a and a luciferase assay identified that PDGFRA was a novel direct target gene of miR-34a. In addition, assays of western blot analyses and quantitative reverse-transcription polymerase chain reaction confirmed that miR-34a decreased PDGFRA mRNA expression and protein levels in colon cancer cells. Assessment of cellular function indicated that miR-34a inhibited colon cancer progression via PDGFRA. These findings demonstrate that miR-34a may act as a negative regulator in colon cancer by targeting PDGFRA.
\end{abstract}

\section{Introduction}

Colon cancer is a common type of cancer worldwide and the treatment of colon cancer is currently problematic. Although there have been marked advances in diagnostic techniques, as well as improvements in treatment modalities, the prognosis of colon cancer remains poor due to its high recurrence (1-3).

Correspondence to: Dr Bo Song, Department of Pathology, Dalian Medical University, 9th West Section, Lvshun South Road, Dalian, Liaoning 116044, P.R. China

E-mail: bo_song2014@163.com

*Contributed equally

Key words: miR-34a, colon cancer, platelet-derived growth factor receptor $\alpha$
Numerous reports show that various signaling pathways are activated during colon cancer progression (1-3); however, the mechanism remains unclear and the identification of novel molecular targets for colon cancer is required.

MicroRNAs (miRNAs) are small RNAs, which negatively regulate gene expression in various types of cell (4). The target genes of miRNAs can be downregulated at a post-transcriptional level. Furthermore, miRNAs acting as tumor inhibitors of onco-miRNAs locate in cancer-associated genomic regions. Their function in the cells depends on their direct target genes (5-7). The primary causes of colon cancer patient mortality are the poor prognosis and metastases. Therefore, it is considered to be critical to establish a novel diagnostic marker and continue to investigate the underlying molecular mechanism of miRNAs for therapeutic application. Emerging evidence demonstrates that a group of miRNAs is involved in the regulation of basic cellular processes in colon cancer, such as cell proliferation, differentiation, apoptosis and metastasis. Previous reports revealed that miR-34a, miR-21, miR-155, miR-499a, miR-99a, miR-101, as well as other miRNAs are involved in colon cancer progression (8-11). Furthermore, an miRNA profile reported that miR-34a is downregulated in colon cancer (9-11).

miR-34a is a known tumor suppressor in various types of cancer, including lung, breast, prostate and liver cancer, as well as in colon cancer (12-18). However, the role of miR-34a in colon cancer requires further investigation. Thus, the aim of the present study was to investigate the underlying effect of miR-34a in colon cancer. miR-34a expression was analyzed in human colon cancer tissues and cells and, using bioinformatics, platelet-derived growth factor receptor $\alpha$ (PDGFRA) was determined to be a target gene of miR-34a. Furthermore, miR-34a was identified to be a tumor suppressor, which negatively regulates the PDGFRA signaling pathway in the progression of colon cancer. The roles of miR-34a in cell proliferation, migration and invasion were also evaluated.

\section{Materials and methods}

Colon cancer samples. Primary colon cancer specimens and normal biopsies were obtained from The First Affiliated Hospital of Dalian Medical University (Dalian, China). A total of 176 colon cancer specimens and normal biopsies were obtained from patients undergoing radical surgery for 
colon cancer at The First Affiliated Hospital (Dalian Medical University, Dalian, China). There were 95 male and 86 female patients (mean age, 57.2-years old). Colon cancer and healthy tissues were confirmed by hematoxylin and eosin staining in the pathology department of The First Affiliated Hospital. The study was approved by the Ethics Committee of The First Affiliated Hospital (Dalian Medical University, Dalian, China) Analyses of tissue samples were performed according to the instructions laid out by the Ethics Committee of the Hospital. All of the patients provided written informed consent.

Cell lines and culture. Colon cancer cells, HT-29, SW620, Lovo and Colo205, were originally obtained from the American Type Culture Collection. The cells were cultured in Dulbecco's modified Eagle's medium (Invitrogen Life Technologies, Carlsbad, CA, USA) supplemented with $10 \%$ fetal bovine serum (Gibco Life Technologies, Carlsbad, CA, USA), $100 \mathrm{U} / \mathrm{ml}$ of penicillin and $100 \mu \mathrm{g} / \mathrm{ml}$ of streptomycin (Sigma-Aldrich, St. Louis, MO, USA) at $37^{\circ} \mathrm{C}$ in a humidified atmosphere of $5 \% \mathrm{CO}_{2}$. Normal colon epithelial cells, NCM460 and FHC, were cultured according to the manufacturer's instructions.

Luciferase assay. A psiCHECK ${ }^{\mathrm{TM}}-2$ Dual-Luciferase miRNA target expression vector was used for the 3'-untranslated region (UTR) luciferase assays (Sangon Biotech, Co., Ltd., Shanghai, China). The target genes of miR-34a were selected on the basis of the following miRNA target database: http://www.microrna.org/microrna/home.do. The 3'-UTR sequence of the PDGFRA gene was amplified using polymerase chain reaction (PCR) and cloned into the $\mathrm{psiCHECK}^{\mathrm{TM}}-2$ vector to produce a wild-type reporter. The following primer sequences were used to amplify the wild-type 3'-UTRs of PDGFRA: Forward, 5'-TCTAGACCGGCCTGAGAAACACTATTT GTG-3' and reverse, 5'-TCTAGAACATGAACAGGGGCA TTCGTAATACA-3'. The mutant reporter construct was generated using the Site-Directed Gene Mutagenesis kit (Beyotime Institute of Biotechnology, Shanghai, China) according to the manufacturer's instructions. There are two binding sites in the PDGFRA 3'-UTR. For one mutant 3'-UTR of PDGFRA, the following primer sequences were used: Forward, 5'-ACT GCCAAAACATTTATGACAAGCTGTATCGCCTCG-3' and reverse, 5'-CGAGGCGATACAGCTTGTCATAAATGT TTTGGCAGT-3'. For the other mutant 3'-UTR of PDGFRA, the following primer sequences were used: Forward, 5'-ACT GCCAAAACATTTATGACAAGCTGTATGGTCGTTTATA TTT-3' and reverse, 5'-AAATATAAACGACCATACAGCTTG TCATAAATGTTTTGGCAGT-3'. For the luciferase assay, a total of 5,000 cells were transfected with miR-34a mimics and the psiCHECK ${ }^{\mathrm{TM}}-2$ Dual-Luciferase miRNA target expression vectors containing the wild-type or the mutant target sequences using Lipofectamine 2000 (Invitrogen Life Technologies). Data are presented as relative firefly luciferase activity normalized to Renilla luciferase activity from the same construct.

Lentiviral infection. The sequence of mature miR-34a was synthesized, amplified and cloned into a GV232-Puro Vector by Genechem Co., Ltd. (Shanghai, China). DNA sequencing was used to identify the correct sequences. Lentivirus mediating miR-34a or its control was produced in $293 \mathrm{~T}$ cells using Lipofectamine 2000 reagent according to the manufacturer's instructions. For transduction, the colon cancer cells were infected with miR-34a or its control and subsequently selected using $1 \mu \mathrm{g} / \mathrm{ml}$ puromycin (Sigma-Aldrich). Lentivirus-mediated silencing of miR-34a was verified by reverse-transcription quantitative (RT-q)PCR analysis.

$R N A$ extraction, isolation and $R T-q P C R$. Total RNA was extracted from the cells and the tissues using TRIzol reagent (Invitrogen Life Technologies). RNA ( $2 \mu \mathrm{g})$ was reverse transcribed to cDNA using a SYBR ${ }^{\circledR}$ Green PCR kit (Takara Biotechnology Co., Ltd., Shiga, Japan). To measure miRNA expression, qPCR was performed for DNA amplification (ABI-Prism; Applied Biosystems Life Technologies, Foster City, CA, USA). The cycling conditions were $10 \mathrm{~min}$ of polymerase activation at $95^{\circ} \mathrm{C}$, followed by 40 cycles at $95^{\circ} \mathrm{C}$ for $15 \mathrm{sec}$ and $60^{\circ} \mathrm{C}$ for $60 \mathrm{sec}$. The relative expression levels were calculated by comparing the cycle threshold values of the samples with those of the references, all data were normalized to glyceraldehyde 3-phosphate dehydrogenase (GAPDH) or the U6 sRNA control.

MTT assay. An MTT assay was conducted to detect cell viability. The colon cancer cells were seeded into 96-well plates following transfection with miRNA or the plasmids. At day 1, 2, 3, 4 and 5, $20 \mu \mathrm{l}$ MTT solution (Sigma-Aldrich) was added to each well (5 mg/ml; 0.5\% MTT) and the cells continued to culture for $4 \mathrm{~h}$. The supernatant was discarded, $150 \mu \mathrm{l}$ dimethyl sulfoxide (Sangon Biotech, Co., Ltd.) was added to each well and the culture plate was agitated at 50-75 x g for $10 \mathrm{~min}$ to ensure crystals were completely dissolved. An ELISA reader was used to measure the absorbance at $570 \mathrm{~nm}$. The MTT assay was performed three times.

Colony forming assay. The colon cancer cells were transfected with miRNA or DNA plasmids and collected following transfection for $24 \mathrm{~h}$. The transfected cells were seeded into 6 -well plates with 200 cells per well, cultured for three days and the nonadherent cells were removed. After 10-14 days, the colonies were dyed with $0.1 \%$ crystal violet (Sigma-Aldrich) and counted.

Western blot analysis. Cells were lysed using radioimmunoprecipitation assay buffer (Santa Cruz Biotechnology, Inc., Dallas, TX, USA) with $1 \mathrm{X}$ protease inhibitor cocktail and total protein was extracted. The concentration of protein was determined using the Bradford assay (Bio-Rad Laboratories, Inc., Philadelphia, PA, USA). SDS-PAGE (12.5\%) was conducted to separate the proteins and the separated proteins were transferred to polyvinylidene difluoride membranes (Merck Millipore, Bedford, MA, USA) at $55 \mathrm{~V}$ for $4 \mathrm{~h}$ at $4^{\circ} \mathrm{C}$. The membranes were blocked in $5 \%$ fat-free milk, incubated in the primary antibodies and then incubated with secondary antibodies for $1 \mathrm{~h}$ at room temperature. PDGFRA (cat. no. 3164), p-Akt (Ser473; cat. no. 4060), Akt (cat. no. 9272) and p21 (cat. no. 2946) primary antibodies were purchased from Cell Signaling Technology, Inc. (Danvers, MA, USA). The dilutions of the above antibodies were all 1:1,000. GAPDH antibody was purchased from Santa Cruz Biotechnology, Inc. (1:5,000). Protein bands were visualized on X-ray film (Sigma-Aldrich) using an enhanced chemiluminescence detection system. 
A

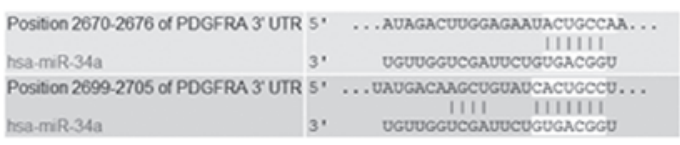

C

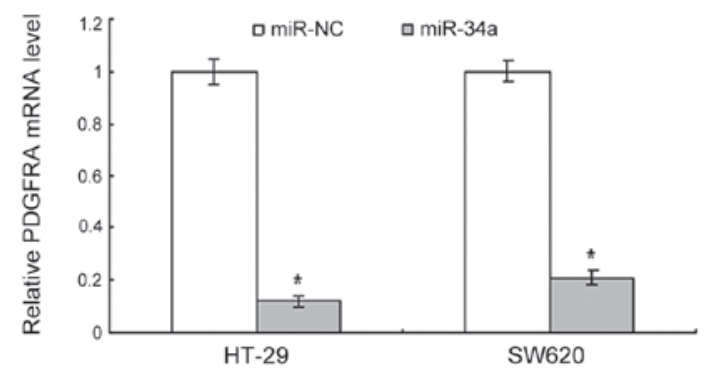

B

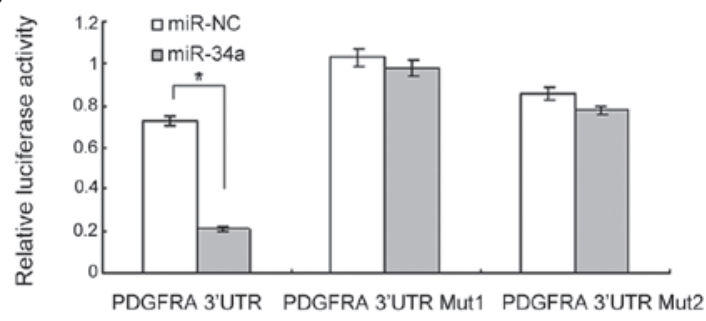

$\mathbf{D}$

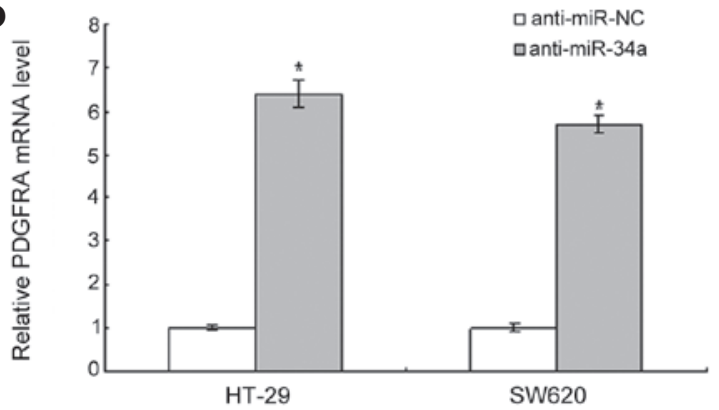

$\mathbf{E}$
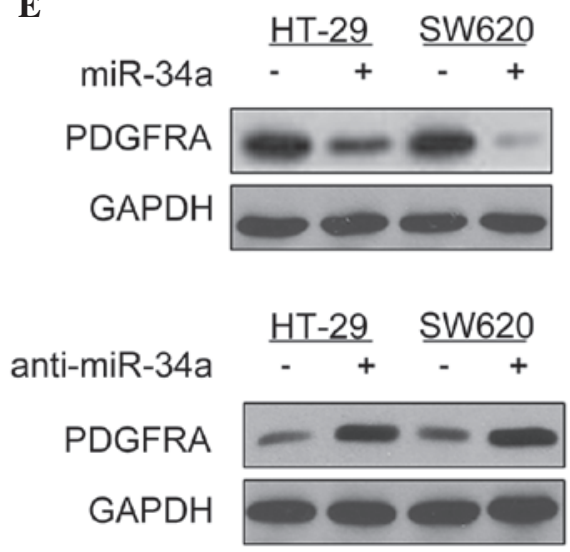

Figure 1. Restoration of miR-34a downregulates PDGFRA expression. (A) The 3'-UTR of the PDGFRA gene contains binding sites for miR-34a according to bioinformatic analysis. (B) miR-34a suppressed the expression of a luciferase reporter gene that was harbouring the $3^{\prime}-\mathrm{UTR}_{\mathrm{T}}$ of PDGFRA. The psiCHECK ${ }^{\mathrm{TM}}-2$ plasmid was modified by adding the human 3'-UTR or the 3'-UTR with mutations in regions complementary to the miR-34a seed regions after the firefly luciferase gene. Cells were transiently co-transfected with miR-34a or the control together with the indicated luciferase constructs, and luciferase activity was analyzed $48 \mathrm{~h}$ subsequently. Data are presented as relative firefly luciferase activity normalized to Renilla luciferase activity from the same construct. (C) miR-34a downregulated PDGFRA mRNA expression levels in colon cancer cells. HT-29 and SW620 cells were infected with LV-miR-34a or the control for $48 \mathrm{~h}$, then collected for qPCR. (D) anti-miR-34a upregulated PDGFRA mRNA expression levels in colon cancer cells. HT-29 and SW620 cells were infected with LV-anti-miR-34a or the control for $48 \mathrm{~h}$, then collected for qRT-PCR. (E) miR-34a downregulated PDGFRA protein levels in HT-29 and SW620 cells. HT-29 and SW620 cells were infected with LV-miR-34a or LV-anti-miR-34a and their control for $48 \mathrm{~h}$, then collected for western blot analysis. The data are presented as means \pm standard deviation collected from three independent experiments. ${ }^{*} \mathrm{P}<0.01$ vs. the control. PDGFRA, platelet-derived growth factor receptor $\alpha$; UTR, untranslated region; qPCR, quantitative polymerase chain reaction; GAPDH, glyceraldehyde 3-phosphate dehydrogenase.

Wound healing assays. Cell migration was assessed by measuring the movement of cells into an acellular area, which was scraped using a $200-\mu 1$ pipette tube; the spread of wound closure was observed after $48 \mathrm{~h}$. Images were obtained to assess the level of migration in each group of transfected cells. Migration was quantified by counting the total number of cells that migrated toward the original wound field.

Migration and invasion assay. Colon cancer cells were transfected with miRNA or DNA plasmids and collected following transfection for $48 \mathrm{~h}$. For the migration assay, $1 \times 10^{5}$ colon cancer cells were plated in the top chamber of the non-coated membrane (24-well insert; pore size, $8 \mu \mathrm{m}$; Corning Costar) and allowed to migrate toward serum-containing medium in the lower chamber. Cells were fixed with methanol after a 24-h incubation and stained with $0.1 \%$ crystal violet $(2 \mathrm{mg} / \mathrm{ml})$. The number of cells invading through the membrane was counted under a light microscope (three random fields per well). For the invasion assay, the steps were the same as the migration assay, however, the membrane was coated with Matrigel (BD Biosciences, San Jose, CA, USA). The number of cells invading through the membrane was counted under a light microscope (magnification, $x 40$; three random fields per well). 
Table I. Association between miRNA expression and clinicopathological features.

\begin{tabular}{|c|c|c|c|}
\hline \multirow[b]{2}{*}{ Characteristic } & \multicolumn{2}{|c|}{ miRNA expression } & \multirow[b]{2}{*}{ P-value } \\
\hline & Low & High & \\
\hline Age (years) & & & 0.054 \\
\hline$\geq 60$ & $52(59.1)$ & $36(40.9)$ & \\
\hline$<60$ & $56(63.6)$ & $32(36.4)$ & \\
\hline Gender & & & 0.204 \\
\hline Male & $54(60.0)$ & $40(44.4)$ & \\
\hline Female & $36(40.0)$ & $50(55.6)$ & \\
\hline Tumor size $(\mathrm{cm})$ & & & 0.280 \\
\hline$\geq 6$ & $40(45.5)$ & $28(31.8)$ & \\
\hline$<6$ & $50(55.6)$ & $58(67.4)$ & \\
\hline Histology grade & & & 0.016 \\
\hline Well & $10(11.1)$ & $0(0)$ & \\
\hline Moderate & $54(60.0)$ & $44(48.9)$ & \\
\hline Poor & $26(28.9)$ & $46(51.1)$ & \\
\hline LNM & & & 0.515 \\
\hline No & $38(42.2)$ & $30(33.3)$ & \\
\hline Yes & $52(57.8)$ & $60(66.7)$ & \\
\hline TNM stage & & & 0.012 \\
\hline I & $1(2.3)$ & $54(60.0)$ & \\
\hline II & $10(11.4)$ & $28(31.1)$ & \\
\hline III & $40(45.5)$ & $6(6.8)$ & \\
\hline IV & $39(40.9)$ & $2(2.2)$ & \\
\hline
\end{tabular}

miRNA, micro RNA; LNM, lymph node metastasis; TNM, tumor-node-metastasis. Colon cancer TNM staging was according to the American Joint Committee on Cancer and International Union Against Cancer.

Statistical analysis. Statistical analysis was conducted using SPSS 15.0 (SPSS, Inc., Chicago, IL, USA). Student's t-test was used to analyze the results, all experiments were performed in triplicate and the data are expressed as means \pm standard deviation. $\mathrm{P}<0.05$ was considered to indicate a statistically significant difference.

\section{Results}

miR-34a expression is correlated with clinicopathological features of colon cancer. Firstly, miR-34a expression was evaluated in 176 primary colon cancer tissue samples and their adjacent non-tumor tissues using RT-qPCR. The colon cancer TNM staging was according to the American Joint Committee on Cancer and International Union Against Cancer (19,20). miR-34a expression in colon cancer tissues was observed to be markedly lower when compared with that of their adjacent non-tumor tissues. The analyzed data revealed that the low expression level of miR-34a in colon cancer tissues was significantly associated with lymph node metastasis and a poor, advanced pT stage, as well as invasion into lymphatic vessels (Table I).
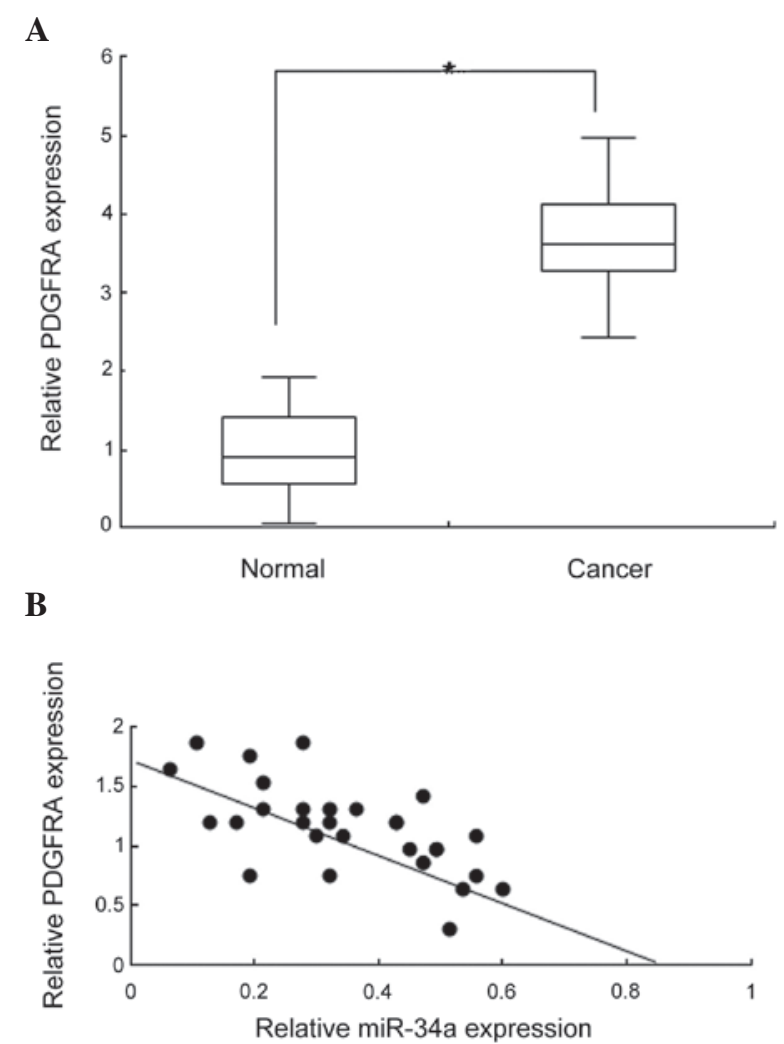

Figure 2. PDGFRA expression levels were inversely correlated with miR-34a expression levels in colon cancer tissue samples. (A) PDGFRA expression levels in 26 paired samples of colon cancer and matched normal tissues were measured by quantitative reverse-transcription polymerase chain reaction. Glyceraldehyde 3-phosphate dehydrogenase served as an internal control. (B) Correlation analysis between PDGFRA mRNA expression levels and miR-34a expression levels in colon cancer tissue samples (Spearman's correlation analysis: $\mathrm{r}=0.422 ; \mathrm{P}<0.05)$. ${ }^{*} \mathrm{P}<0.05$ vs. the control.

PDGFRA is a novel target gene of miR-34a in colon cancer cells. miR-34a exerts a suppressive role in various types of cancer, however, its target gene has not been fully elucidated. Bioinformatic analysis using TargetScan (http://www. targetscan.org/vert_61/) revealed that PDGFRA was directly suppressed by miR-34a (Fig. 1A). As shown in Fig. 1B, the luciferase activity of PDGFRA in 293T cells was significantly lower than in the control cells $(\mathrm{P}<0.01)$. The luciferase activity of the cells with the mutated 3'-UTR of PDGFRA was not changed significantly when compared with the control. Subsequently, whether miR-34a regulates endogenous PDGFRA expression in the cells was examined. In HT-29 and SW620 cells, compared with the control, endogenous PDGFRA mRNA expression (Fig. 1C and D) and protein levels (Fig. 1E) were downregulated in the cells with LV-miR-34a infection and upregulated with anti-miR-34a infection.

PDGFRA expression is negatively associated with $\mathrm{miR}-34$ a expression in colon cancer tissues. To identify the association between the expression of PDGFRA and miR-34a in colon cancer tissue, the expression of PDGFRA in 26 pairs of colon cancer tissue and normal control samples was detected using qRT-qPCR. PDGFRA expression was observed to increase markedly in the cancer tissues when compared with the normal tissues (Fig. 2A; $\mathrm{P}<0.05$ ). Analysis of the data demonstrated that 
A

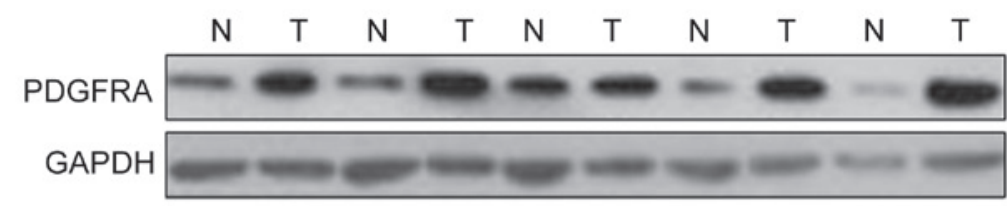

B

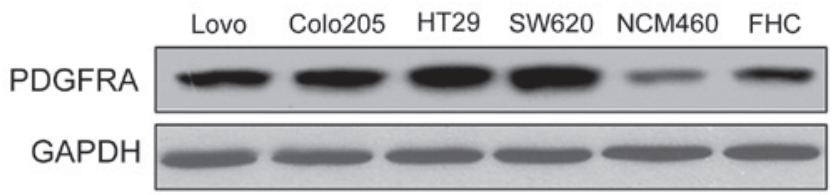

C
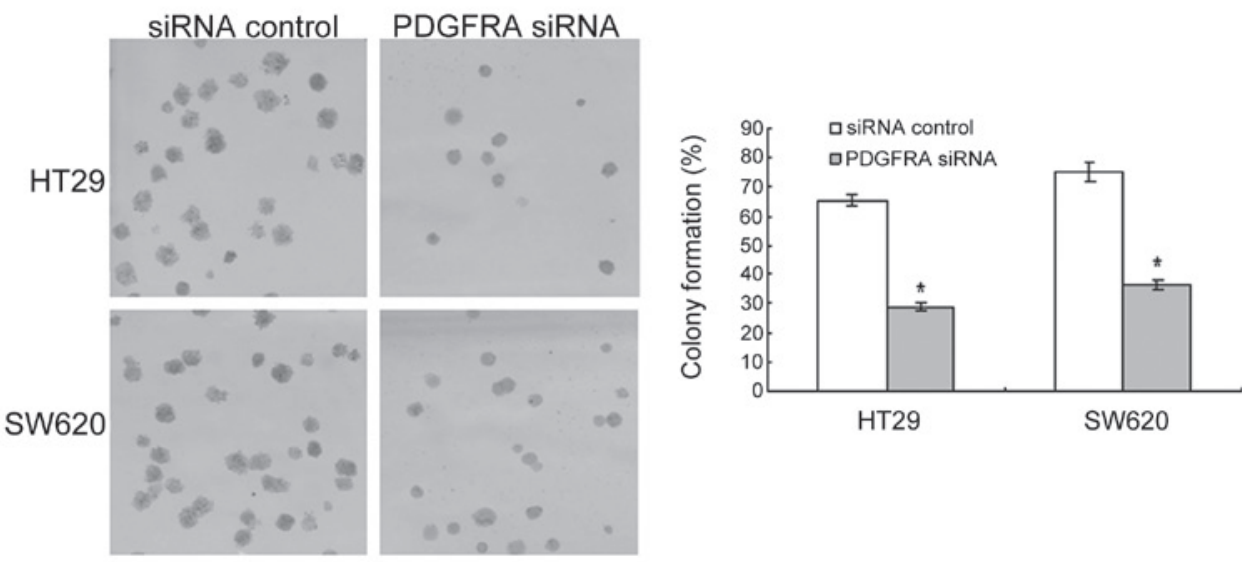

D
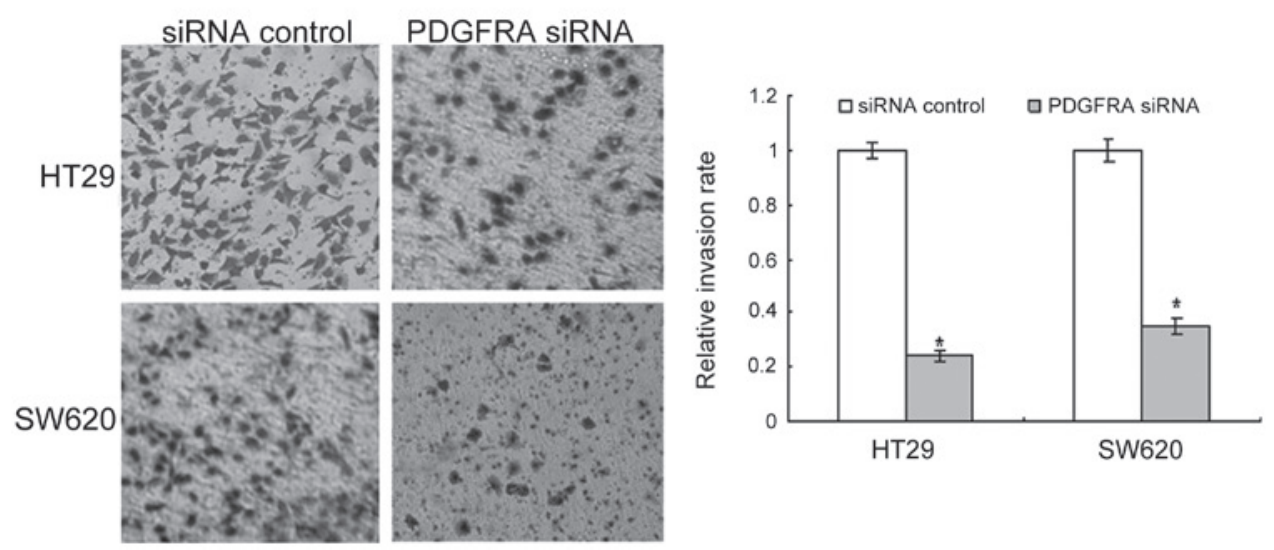

Figure 3. Overexpression of PDGFRA in human colon cancer tissue samples and cell lines. (A) PDGFRA expression was upregulated significantly in colon cancer tissue samples (lane T) compared with normal tissue samples (lane N). (B) The relative expression of PDGFRA in five colon cancer cell lines and the normal colon cell line, FHC, was examined by western blot analysis. (C) Inhibition of PDGFRA expression suppressed cell proliferation in HT-29 and SW620 cells. (D) Downregulation of PDGFRA expression inhibited colon cancer cell invasion. HT-29 and SW620 cells were transfected with PDGFRA siRNA or its control and invasion was detected using a Transwell system. The data are presented as means \pm standard deviation of three independent experiments. ${ }^{*} \mathrm{P}<0.01$ vs. the control. PDGFRA, platelet-derived growth factor receptor $\alpha$; GAPDH, glyceraldehyde 3-phosphate dehydrogenase; siRNA, small interfering RNA.

PDGFRA was negatively associated with miR-34a expression in the majority of tissues (Fig. 2B). These data further indicated that PDGFRA was a target of miR-34a in colon cancer.

PDGFRA increases human colon cancer cell proliferation and metastasis. Previous reports demonstrated that PDGFRA is overexpressed in colon cancer, however, its role has not been determined. To investigate the possible role of PDGFRA in colon cancer cells, the PDGFRA protein was examined in colon cancer cells. As shown in Fig. 3A, PDGFRA expression in colon cancer tissue samples was higher than that in adjacent normal tissue samples. Similarly, PDGFRA was markedly higher in human colon cancer cells than in the normal colon cells (Fig. 3B). These results provide initial evidence that PDGFRA may exert a tumor-suppressing role in the development of human colon cancer. Furthermore, it was noted that cell proliferation and metastasis were decreased in colon cancer cells exhibiting PDGFRA inhibition (Fig. 3C and D).

miR-34a inhibits colon cancer progression by targeting PDGFRA and its signaling pathway. The above-mentioned data indicate that PDGFRA is a target gene of miR-34a. In the present study, elucidating whether miR-34a regulates biological behavior, such as proliferation, apoptosis and metastasis was investigated. PDGFRA overexpression was used to assess whether miR-34a inhibited HT-29 cell metastasis. The result revealed that miR-34a 
A

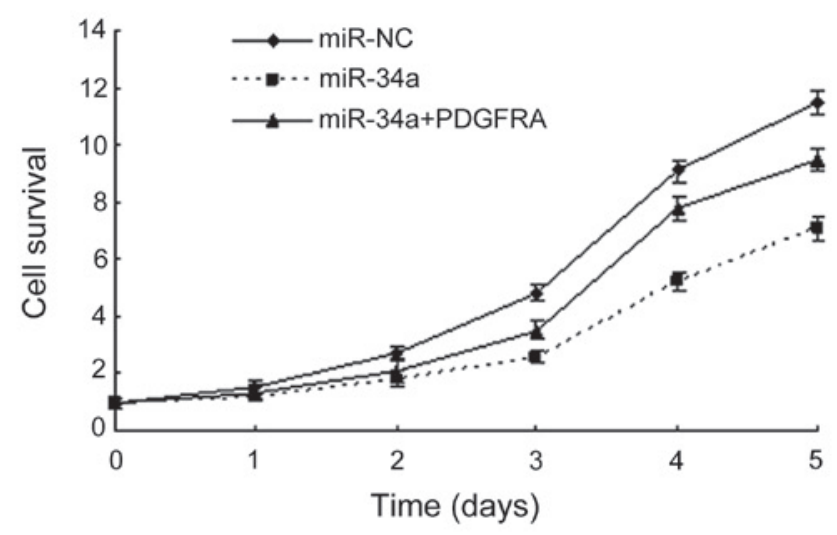

B
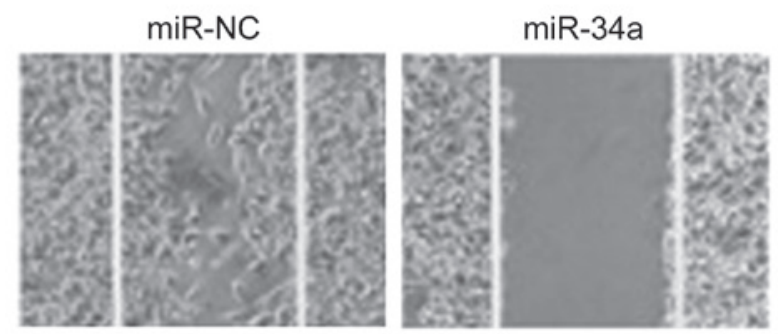

miR-34a+PDGFRA

C
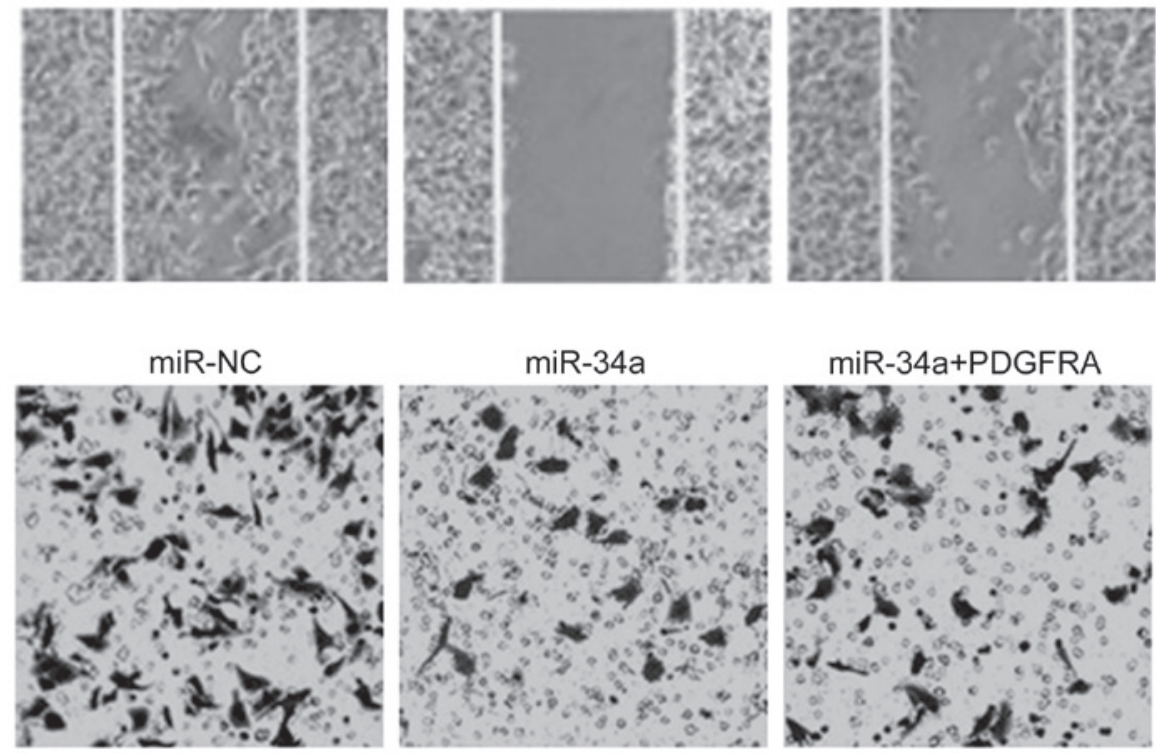

D

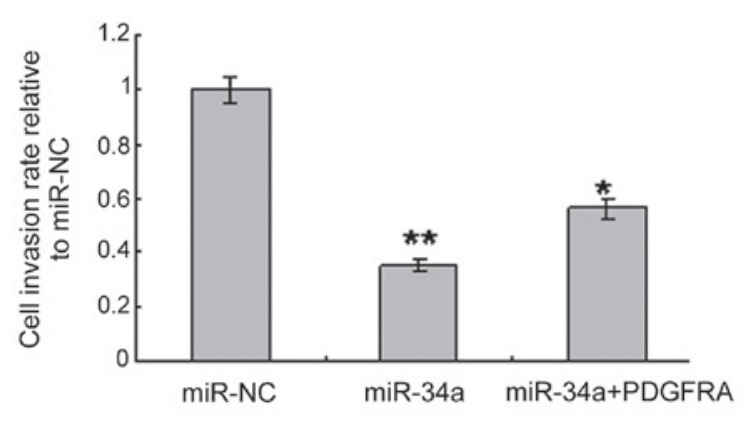

$\mathbf{E}$

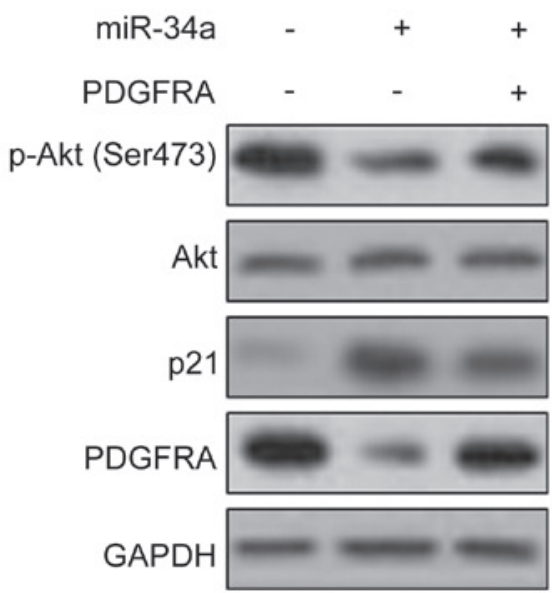

Figure 4. miR-34a inhibits progression of colon cancer-targeting PDGFRA. (A) miR-34a expression inhibited proliferation of HT-29 cells exhibiting PDGFRA overexpression. HT-29 cells were infected with LV-miR-34a or PDGFRA or the control, and cell proliferation at days 0-5 was analyzed by MTT assay. (B) miR-34a expression decreased migration of HT-29 cells with PDGFRA overexpression. (C) miR-34a expression decreased invasion of HT-29 cells with PDGFRA overexpression. (D) Quantification of the extent of cell invasion. (E) miR-34a inhibited the PDGFRA signaling pathway in HT-29 cells. HT-29 cells were infected with LV-miR-34a or PDGFRA or the control, and total protein was extracted for western blot analysis. "P<0.05 vs. the control. " P $<0.01$ vs. the control. PDGFRA, platelet-derived growth factor receptor $\alpha$; GAPDH, glyceraldehyde 3-phosphate dehydrogenase; miRNA, micro RNA; NC, negative control; p, phosphorylated.

expression in the cells inhibited proliferation of HT-29 cells with or without PDGFRA overexpression (Fig.4A). The migration and invasion of the cells increased with PDGFRA expression, and when the cells were infected with LV-miR-34a, migration and invasion were decreased (Fig. 4B-D). As the PDGFRA-mediated signaling pathway is an important pathway involved in cancer 
development, various proteins and downstream proteins in the signaling pathway, such as Akt, were detected and observed to be decreased by miR-34a overexpression (Fig. 4E).

\section{Discussion}

Previous reports demonstrated that miR-34a is involved in colon cancer progression, including inducing apoptosis, and inhibiting cell proliferation and metastasis. However, the role of miR-34a requires further research. In the present study, miR-34a was found to be downregulated in colon cell lines and tissues, and has an important role in metastasis by targeting PDGFRA.

Firstly, the results from the clinical data indicated that miR-34a is associated with the colon cancer stage and metastasis, which indicates that miR-34a is involved in colon cancer cell metastasis. In HT-29 and SW620 colon cancer cells, restoration of miR-34a expression promoted cell growth, and decreased cell migration and invasion (Fig. 3). This indicated that a reduced expression level of miR-34a in colon cancer cells may facilitate cell invasion and metastasis of colon cancer. In addition, PDGFRA expression was observed to be negatively correlated with miR-34a expression in colon cancer cells and tissues.

Using bioinformatic prediction, PDGFRA was identified to be the direct target of miR-34a. Furthermore, overexpression of miR-34a decreased the expression levels of PDGFRA protein and mRNA, indicating that PDGFRA is a downstream gene of miR-34a in colon cancer cells. However, miR-34a in colon cancer may target other genes. It is well known that there are molecules influencing miR-34a, which are involved in colon cancer progression and growth, as well as other functions $(12,13)$.

PDGFRA is overexpressed in various malignancies and is crucial in promoting cell proliferation and metastasis (21-25). In the present study, miR-34a expression was observed to be negatively correlated with PDGFRA. Furthermore, the present results indicate that miR-34a downregulates PDGFRA expression by directly targeting the PDGFRA 3'-UTR. Therefore, it may be possible to use miRNA to target PDGFRA during cancer therapy. However, expression of miRNA or its target genes is influenced by multiple factors, such as epigenetic modification, including DNA methylation, histone modification, chromosome deficiency and transcriptional regulation $(4,5)$. Thus, the underlying mechanisms of the repression of miRNA require further investigation.

In conclusion, the present study verified that the deregulated expression of miR-34a is associated with a poor prognosis and an aggressive phenotype of colon cancer. Furthermore, the findings indicate that miR-34a is significantly involved in the regulation of colonic malignant behavior, including cell proliferation and invasion, by directly targeting PDGFRA. Therefore, miR-34a may serve as a potential prognostic biomarker and as a therapeutic molecule for the treatment of colon cancer.

\section{Acknowledgements}

The present study was supported by grants from the Joint Funds of the Natural Science Foundation of Liaoning Province (grant no. 2013023024), the National Natural Science Foundation of China (grant no. 81172052) and the Yingcai Program of Dalian Medical University.

\section{References}

1. Weitz J, Koch M, Debus J, Höhler T, Galle PR and Büchler MW: Colorectal cancer. Lancet 365: 153-165, 2005.

2. Dyzmann-Sroka A and Malicki J: Cancer incidence and mortality in the greater Poland region-analysis of the year 2010 and future trends. Rep Pract Oncol Radiother 19: 296-300, 2014.

3. Swiderska M, Choromańska B, Dąbrowska E, Konarzewska-Duchnowska E, Choromańska K, Szczurko G, Myśliwiec P, Dadan J, Ladny JR and Zwierz K: The diagnostics of colorectal cancer. Contemp Oncol (Pozn) 18: 1-6, 2014.

4. Dalmay T: MicroRNAs and cancer. J Intern Med 263: 366-375, 2008.

5. Calin GA and Croce CM: MicroRNA signatures in human cancers. Nat Rev Cancer 6: 857-866, 2006.

6. Esquela-Kerscher A and Slack FJ: Oncomirs-microRNAs with a role in cancer. Nat Rev Cancer 6: 259-269, 2006.

7. Negrini M, Ferracin M, Sabbioni S and Croce CM: MicroRNAs in human cancer: From research to therapy. J Cell Sci 120: 1833-1840, 2007.

8. Cho WC: OncomiRs: the discovery and progress of microRNAs in cancers. Mol Cancer 6: 60, 2007.

9. Tokarz P and Blasiak J: The role of microRNA in metastatic colorectal cancer and its significance in cancer prognosis and treatment. Acta Biochim Pol 59: 467-74, 2012.

10. Liu C and Tang DG: MicroRNA regulation of cancer stem cells. Cancer Res 71: 5950-5954, 2011.

11. Lai X, Wolkenhauer O and Vera J: Modeling miRNA regulation in cancer signaling systems: miR-34a regulation of the p53/Sirt1 signaling module. Methods Mol Biol 880: 87-108, 2012.

12. Chen F and Hu SJ: Effect of microRNA-34a in cell cycle, differentiation and apoptosis: A review. J Biochem Mol Toxicol 26: 79-86, 2012.

13. Hermeking H: The miR-34 family in cancer and apoptosis. Cell Death Differ 17: 193-199, 2010.

14. Ma Y, Bao-Han W, Lv X, Su Y, Zhao X, Yin Y, Zhang X, Zhou Z, MacNaughton WK and Wang H: MicroRNA-34a mediates the autocrine signaling of PAR2-activating proteinase and its role in colonic cancer cell proliferation. PLoS One 8: e72383, 2013.

15. Bu P, Chen KY, Chen JH, Wang L, Walters J, Shin YJ, Goerger JP, Sun J, Witherspoon M, Rakhilin N, et al: A microRNA miR-34a-regulated bimodal switch targets Notch in colon cancer stem cells. Cell Stem Cell 12: 602-615, 2013.

16. Winton DJ: miR-34a sets the 'sweet spot' for notch in colorectal cancer stem cells. Cell Stem Cell 12: 499-501, 2013.

17. Yamakuchi M, Ferlito M and Lowenstein CJ: miR-34a repression of SIRT1 regulates apoptosis. Proc Natl Acad Sci USA 105: 13421-13426, 2008.

18. Tazawa $H$, Tsuchiya $N$, Izumiya $M$ and Nakagama $H$ : Tumor-suppressive miR-34a induces senescence-like growth arrest through modulation of the E2F pathway in human colon cancer cells. Proc Natl Acad Sci USA 104: 15472-15477, 2007.

19. Rudy DR and Zdon MJ: Update on colorectal cancer. Am Fam Physician 61: 1759-1770, 1773-1774, 2000.

20. Jass JR and Morson BC: Reporting colorectal cancer. J Clin Pathol 40: 1016-1023, 1987.

21. Zhu Y, Wang Y, Guan B, Rao Q, Wang J, Ma H, Zhang Z and Zhou X: C-kit and PDGFRA gene mutations in triple negative breast cancer. Int J Clin Exp Pathol 7: 4280-4285, 2014.

22. Stock AM, Hahn SA, Troost G, Niggemann B, Zänker KS and Entschladen F: Induction of pancreatic cancer cell migration by an autocrine epidermal growth factor receptor activation. Exp Cell Res 326: 307-314, 2014.

23. Demoulin JB and Essaghir A: PDGF receptor signaling networks in normal and cancer cells. Cytokine Growth Factor Rev 25: 273-283, 2014

24. Hayes BJ, Riehle KJ, Shimizu-Albergine M, Bauer RL, Hudkins KL, Johansson F, Yeh MM, Mahoney WM Jr, Yeung RS, Campbell JS, et al: Activation of platelet-derived growth factor receptor alpha contributes to liver fibrosis. PLoS One 9: e92925, 2014.

25. Roh JW, Huang J, Hu W, Yang X, Jennings NB, Sehgal V, Sohn BH, Han HD, Lee SJ, Thanapprapasr D, et al: Biologic effects of platelet-derived growth factor receptor $\alpha$ blockade in uterine cancer. Clin Cancer Res 20: 2740-2750, 2014. 